\title{
La représentation des petites filles dans les romans pour la jeunesse: de Sophie de Ségur à Élise Turcotte
}

\author{
Lucie Guillemette
}

This study examines Élise Turcotte's trilogy for young readers. This series introduces a little girl named Annette and is aimed at readers seven years old and older. The author studies the discursive practices that explain the little girl's relationship to her environment and to the world of knowledge in a time when feminist thought shaped the collective imaginary and transformed social roles. Specifically, it looks at feminine subjectivity, which in the context of an enunciation that addresses a young readership articulates a voice able to deconstruct modern meta-discourses by investing the adults' objectifying discourse. The little girl, engaged in the process of writing, defines herself and questions the systems of beliefs emanating from her family setting.

Nombreux sont les spécialistes ${ }^{i}$ qui se demandent si le mouvement féministe a modifié de façon significative la perception que les fillettes ont d'elles-mêmes en général, et la représentation que l'on en fait dans les œuvres littéraires, en particulier. Il y a trente ans, les travaux de Elena G. Belotti ont corroboré l'hypothèse stipulant que les conditionnements sociaux ont une incidence sur la formation du rôle féminin dans la petite enfance. Selon l'auteure, " il est possible de modifier les causes sociales et culturelles qui seraient à l'origine des différences entre les sexes » (Belotti 10). Comme l'ont par la suite montré les études sur le genre et le sexe social, la féminité se réduit à un artefact dans la mesure où l'éducation des filles est inféodée aux normes d'une société patriarcale. Qu'en est-il aujourd'hui des fillettes dont la formation tend à s'écarter des modèles masculins et des rapports de pouvoir qu'ils sous-tendent, et qui revendiquent une identité fondée sur l'autodétermination et l'expérience? Les petites filles sont-elles en mesure d'articuler une parole féminine désirant coûte que coûte désavouer l'idéologie totalisante du patriarcat?

Si l'on veut apporter des éléments de réponse à ces interrogations, l'étude d'une production romanesque écrite à l'intention de la jeunesse et, par conséquent, surdéterminée par un jeune destinataire, peut s'avérer éclairante. Alors qu'il s'inscrit dans un appareil de communication dont l'allocutaire est précisément un enfant, le texte littéraire qui représente une jeune héroïne transpose une réalité sociale donnée. Outil pédagogique, le roman destiné à la jeunesse constitue certes une voie de transmission des valeurs et des modèles culturels. Dans cette foulée, Sylvie Cromer et Adela Turin soulignent que « [les] albums sont la première lecture de jeunesse et [que] les enfants garderont longtemps en mémoire leurs images, qu'ils auront longuement regardées » (223). 
Si le périple en forêt d'un petit chaperon rouge combien vulnérable et la sortie au bal d'une Cendrillon médusée au coup de minuit ont contribué à valoriser des modèles féminins d'obéissance et de soumission au sein des contes traditionnels, des petites filles aspirant à l'autonomie et à une liberté d'action campent les romans contemporains pour la jeunesse, publiés depuis une quinzaine d'années au Québec. Que l'on songe à Sophie qui veut devenir gardien de but et joindre une équipe de hockey masculine (Leblanc 1991), à Méli Mélo qui préfére la compagnie d'un monstre sur une boîte de céréales à celle de son père (Hébert 1988), à Marie-Cléo surnommée « Marie la chipie » en raison de l'ascendant qu'elle a sur son frère aîné (Demers 1997), à Cassiopée qui fait une fugue à New York pour y découvrir, outre un premier amour, un monde insoupçonné de connaissances littéraires et scientifiques (Marineau 1988), à Myralie dont la passion pour la botanique en fait l'auteure d'un herbier et une alliée de la flore laurentienne (Boisvert 1999). Autant de jeunes protagonistes qui se retrouvent à divers degrés projetées dans le feu de l'action et dont les aventures font éclater les cadres d'une éducation axée sur la transmission des valeurs traditionnelles, héritées d'un mythe judéo-chrétien postulant l'infériorité de la femme. Depuis les années 1960, force est de constater que les menées d'un féminisme favorisant l'égalité des sexes dans la sphère dite publique ont engendré une profonde réflexion à l'endroit de l'éducation des filles et, par le fait même, de la répartition des rôles sociaux chez les hommes et les femmes. De tels changements ont fait en sorte que le sujet féminin s'est doté d'outils pour récuser le conditionnement social et l'objectivation qui en découle ${ }^{\mathrm{ii}}$, bien qu'il reste encore à accomplir. Aussi les images des fillettes que proposent les textes sollicitant un jeune lectorat comportent des faits de lutte et de désobéissance qui tranchent résolument avec les modèles de passivité présentés dans les romans de la comtesse de Ségur au XIX siècle. Les « petites filles modèles " qui ont fait la gloire de ces classiques pour la jeunesse souscrivent en tous points aux schèmes d'une idéologie patriarcale visant à faire des fillettes des épouses soumises et des mères dévouées. Dans L'Éducation des petites filles chez la comtesse de Ségur, Marie-Christine Vinson parle pour sa part d'un " enfermement idéologique » (14), alors qu'elle décrit les pouvoirs d'oppression au cour de textes où les activités des femmes s'effectuent dans les limites du travail domestique. Sous la tutelle d'une mère " enfermée » au château et dont la mission est de perpétuer le discours masculin, les petites filles qui évoluent à travers l'œuvre ségurienne n'ont d'autre choix que d'obéir. « Normaliser et punir ", tel est le mot d'ordre. Tout se passe comme si les fillettes livrées au regard disciplinaire d'une société imposant ses normes et ses stéréotypes vivaient dans un milieu carcéraliii. Qu'en est-il alors des petites filles présentées dans les œuvres à l'aube du $\mathrm{XXI}^{\mathrm{e}}$ siècle ?

Dans le cadre de la présente étude, une trilogie dont la romancière et poète Élise Turcotte est l'auteure fera l'objet de mon investigation. Il s'agit d'une série destinée à un public âgé de sept ans et plus et qui paraît à partir de la fin des années 1990 aux éditions de la Courte échelle, une maison dont le catalogue est très populaire auprès des jeunes lecteurs québécois ${ }^{\mathrm{iv}}$. L'histoire mettant en scène une petite fille dont le prénom est Annette s'organise au sein d'une production mini- 
romanesque qui se compose de trois ouvrages: Les Cahiers d'Annette (1998), La Leçon d'Annette (1999) et Annette et le vol de nuit (2000). Symptomatiques de l'éveil d'une subjectivité, les récits relatés à la première personne sont évocateurs des progrès accomplis quant à la figuration des fillettes à travers le discours littéraire et ce, depuis la publication de « la trilogie des Fleurville » qui regroupe les ouvrages suivants: Les Petites filles modèles (1858), Les Vacances (1859) et Les Malheurs de Sophie (1860). Autobiographiques, ces textes témoignent sous plusieurs aspects de l'expérience familiale de Sophie de Ségur, née Rostopchine, une femme issue de la classe aristocratique de Saint Pétersbourg en Russie, puis convertie au catholicisme peu de temps avant d'épouser Eugène de Ségur. Accordant une place prépondérante à l'éducation des fillettes grandissant dans la France du Second Empire, ces romans dont l'action se situe dans la région de 1'Orne, en Normandie, se font le reflet d'une époque où l'éducation morale assujettit sans vergogne le sexe féminin, de sorte qu'une narration omnisciente éclipse la voix des petites filles. À bien des égards, la trilogie de Turcotte établit un régime d'oppositions avec l'œuvre ségurienne dans la mesure où l'héroïne, contrairement aux vertueuses Camille et Madeleine v , prône la désobéissance et met en question l'autorité parentale. Afin de décrire les liens qui se tissent entre des ouvrages destinés à la jeunesse, que plus d'un siècle sépare, je me propose d'examiner les pratiques discursives qui éclairent le rapport de la jeune Annette avec son entourage et le monde de la connaissance à une ère où la pensée féministe a façonné des imaginaires et modifié les rôles sociaux. Il sera ainsi question d'une subjectivité féminine qui, dans le contexte d'une énonciation convoquant un jeune lectorat, articule une voix susceptible de déconstruire les " métarécits ${ }^{\mathrm{vi}}$ " modernes investissant le discours objectivant des adultes. Engagée dans un processus d'écriture, la fillette énonce un moi en devenir tout en mettant en question les systèmes de croyances émanant du milieu familial.

\section{La petite fille et l'écriture : une quête identitaire}

Éloquente dans l'apprentissage, Annette est l'unique narratrice des événements dont elle est l'héroïne dans la série de Turcotte. Âgée de huit ans, lorsque s'amorce le premier tome, la jeune protagoniste prend en charge le récit de ses aventures qu'elle commente à l'intérieur des carnets: « Ce que j'aime, c'est écrire toutes sortes de choses dans toutes sortes de petits cahiers. [...] Ce ne sont pas des aventures abracadabrantes. Plutôt des petits riens qui existent dans la vie, ou dans les livres, ou qui m'arrivent à moi, ou à mon frère, ou à l'un ou l'autre de mes nombreux parents proches ou éloignés » (Turcotte 1998, 10-11). À travers le premier roman dont le titre est Les Cahiers d'Annette, l'héroïne développe une réflexion sur sa pratique scripturale alors qu'elle scrute le contexte dans lequel surviennent les mots. Sensible à la matière sonore des signes du langage, Annette réagit aux vocables qui se greffent au discours: « Ce mot me plaît: capharnaüm! Il donne le cafard comme on dit dans les livres de ma mère. Pas trop. Juste assez pour se sentir un peu bizarre. Et j'aime me sentir un peu bizarre » (35). Manifestement, la mise en rapport de signifiants dont les particules phoniques sont voisines suscite une gamme d'émotions chez l'écrivaine en herbe qui confère aux mots superposés 
des significations originales. En vertu d'une activité intense d'écriture, le je féminin accuse les traits d'une conscience narrante dont le moi s'énonce dans le quotidien sans chercher à gloser sur des événements à caractère sensationnel. D'ailleurs, dès l'incipit le lecteur comprend que la fillette préfère la tranquillité de sa chambre, propice au recueillement imposé par l'écriture, aux promenades dans les centres commerciaux en compagnie de sa mère : "Mais les sorties, ça ne m'intéresse pas » (9). Aussi la culture de l'écrit qui prévaut dans le contexte familial de l'héroïne favorise-t-elle la rédaction d'un journal personnel ${ }^{\text {vii }}$. Vivant auprès d'une femme passionnée par la lecture et d'un garçon âgé de six ans qui réussit fort bien à l'école, Annette rédige des textes à saveur autobiographique que sa mère qualifie de chroniques. Comme l'indique le dictionnaire, la chronique consiste en un " recueil de faits historiques, rapportés dans l'ordre de leur succession " (Le Petit Robert 312). On comprend ainsi que les écrits de la petite fille pourraient se rapprocher des mémoires et du récit. C'est pourquoi, au dire de la fillette, les cahiers qu'elle complète prennent la forme de " petits riens qui existent dans la vie " (Turcotte 1998, 11).

Quoique ses textes cherchent à refléter l'existence dans ce qu'elle comporte de linéaire et prosaïque, le sujet féminin se plaint du fait qu'« il ne [lui] arrive jamais rien » (25). De fait, la narratrice envie sa cousine qui vit aux Seychelles, sous un climat tropical, et qui s'amuse avec les tortues géantes dont on lui a fait cadeau. Captivée par les figures de l'ailleurs et de l'exotisme, la fillette considère qu'elle mène auprès de sa famille une vie sans couleur et sans mystère: «Moi, je voudrais vivre des expériences incroyables, sauf qu'ici notre vie est banale » (12). De plus, le personnage s'insurge contre le fait qu'il ne possède pas de « jardin secret », à savoir des objets qui authentifieraient et marqueraient de façon spécifique son individualité. À la suite de sa rencontre avec des jumelles, Annette se rend à leur domicile et demeure mystifiée par la présence d'une boîte sur laquelle apparaissent des ballerines. Il s'agit d'un présent d'autant plus énigmatique que les gamines ne peuvent l'ouvrir avant l'âge de dix ans. Déjà passablement éprouvée par une existence sans histoire et une physionomie aux traits quelconques, l'héroïne déplore le fait qu'on ne lui ait jamais offert de cadeau analogue à la « boîte cachant un mystère » (46), puisqu'《 [elle] est comme tout le monde » (48). À l'inverse de la jumelle qui se prénomme Cybèle et qui possède des yeux de couleur différente, le physique d'Annette, à son grand désarroi, ne comporte aucun signe particulier: " J'ai des cheveux châtains, deux yeux bleus, et je suis laide quand je souris! » (51). Pour ajouter au malheur de la fillette se plaignant d'être affublée d'un prénom qui demeure à ses yeux « le pire qui existe dans le dictionnaire des prénoms » $(51)$, « Cybèle » qui renvoie à une déesse de la mythologie reste évocateur: " Déesse de la terre, fille du ciel [...], Cybèle symbolise l'énergie enfermée dans la terre. [...] Elle est la source primordiale de toute fécondité » (Chevalier et Gheerbrant 330). Comparativement aux objets qu'on trouve chez ses nouvelles amies, les cahiers lui semblent désormais sans panache étant donné qu'ils s'attardent à des futilités: « les gardiens de chaussures, les histoires de princesses japonaises, les recettes de bonbons, les codes secrets, les jeux vidéo que je veux inventer, la liste de qualités et de défauts des 
gars de ma classe... [sic] » (Turcotte 1998, 46). Persuadée que ses écrits se rapportent à « des choses stupides » et insignifiantes, la gamine suspend la rédaction: « Je prends tous mes cahiers et descends à la cuisine pour les jeter à la poubelle » (46). Tout se passe comme si l'auteure des chroniques percevait l'absurdité d'une existence dépourvue de faits saillants dans la mesure où elle souhaite façonner son moi et lui donner du relief. Semblable revendication d'unicité atteint son paroxysme lors de la scène où l'on célèbre l'anniversaire d'Annette à qui la mère fait présent de deux petites tortues. Malheureuse face à des animaux qu'elle n'est pas certaine de pouvoir aimer, la petite fille reprend l'écriture des cahiers pour oublier son chagrin et exprimer ses états d'âme.

À l'exemple de madame de Fleurville qui enseigne les bonnes manières à ses filles dans les romans de Ségur, la mère d'Annette préconise la gentillesse en tout temps et en tout lieu, notamment lorsque l'héroïne côtoie des camarades de jeu. Curieusement, l'amitié ne revêt pas d'attrait particulier aux yeux de la protagoniste qui supporte mal une compagnie prolongée: "Chaque fois que Véronique vient jouer avec moi, au bout d'une heure ou deux j'ai envie qu'elle parte. Alors je lui propose de s'en aller » (26). En ces occasions, la mère s'empresse de rappeler à sa fille les règles de la politesse et de la courtoisie, indispensables au bon fonctionnement d'une société dite civilisée. Consciente de ses comportements d'enfant rebelle, la narratrice reproduit les réactions de l'adulte et son sens des convenances: " "Sois gentille." "Ne dis pas ça." [...] "Ce n'est pas poli. Ça ne se dit pas." Voilà ce qu'elle me répète » (6). De toute évidence, la gamine n'a que faire des discours qui mettent un frein à sa volonté et entravent sa liberté d'action. Rien d'étonnant si le discours maternel truffé d'impératifs et de prescriptions la rebute: "C'est toujours pareil avec elle : "il faut". Il faut des amis, il faut lire, il faut sortir, il faut sourire... » (55). De fait, la fillette ne se gêne pas pour émettre des commentaires à l'endroit des adultes dont elle questionne ouvertement le goût. Au moment où elle croise à nouveau les jumelles, elle constate que celles-ci sont vêtues de la même manière: « Les parents n'ont pas de bon sens. Ce n'est plus la mode d'habiller des jumelles de façon identique, tout le monde le sait! " (33). À la lumière de ces remarques, force est de constater que le personnage féminin ne craint pas de critiquer les adultes et leur mode de vie.

À l'encontre des petites filles modèles respectant les principes d'une morale religieuse véhiculés par les adultes, Annette se soustrait à la morale de l'aînée, elle qui est davantage intéressée à comprendre l'effet du langage et son accumulation de signifiants dans le flux perceptif. Ainsi, la fillette s'adjoint la mère pour conclure une expérience faisant appel au prénom de sa meilleure amie: "Je voulais qu'elle prononce les mots : "Véronique est morte" pour savoir ce que ça me ferait » (23-24). Non seulement la gamine se réfère-t-elle à la mort comme une expérience parmi tant d'autres puisque « après la mort on devient du compost $"(25-26)$, mais encore cherche-t-elle à s'en approprier la signification à travers le langage. Lorsque la maman évoque la présence des anges pour rassurer le petit frère s'interrogeant sur la vie après la mort, la fillette réfute toute spéculation métaphysique à portée spirituelle. En revanche, elle insiste sur la détérioration du corps humain au cœur de la nature, appelé à « [nourrir] les fleurs et les 
arbres » (26). Aux explications à connotation religieuse fournies par la mère, Annette oppose des considérations matérielles rappelant le destin excrémentiel du corps après avoir rendu l'âme. Pareille philosophie matérialiste ne peut qu'éloigner la petite fille des schèmes manichéens du bien et du mal qui ont alimenté la trame narrative des romans séguriens.

\section{La petite fille et les lieux de désobéissance : l'apprentissage de la différence}

Tandis que les personnages de la comtesse de Ségur font l'apprentissage de l'ordre et de l'obéissance en vue de devenir des épouses parfaites, Annette entreprend de donner une leçon de désobéissance à son frère qu'elle juge trop docile: « Mais il est parfait ! Doux comme un agneau, dit ma mère " (10). Dans La leçon d'Annette, l'héroïne, âgée de neuf ans, initie le jeune Raphaël à l'insubordination dans la mesure où elle ne conçoit pas qu'un enfant de six ans soit si raisonnable: « Il a de bonnes notes à l'école. Il fait tout ce qu'on lui demande. Il rapporte même ses mauvais coups " (7). Loin de fulminer contre l'école, le jeune garçon rappelle immanquablement sa mère à l'ordre lorsque vient le temps de respecter les horaires et de faire les devoirs. Bref, il s'agit d'un garçon modèle, " plus raisonnable que ses parents » (16). De tels comportements sont inadmissibles aux yeux de la protagoniste pour qui liberté rime avec rébellion et turbulence. Outrée de voir son frère se conformer indûment aux prescriptions des adultes, la petite fille décide de mettre un terme à ces excès de naïveté et concocte un plan en vue d'inculquer quelques rudiments de désobéissance à son cadet. Autrement dit, la narratrice veut déprogrammer son frère et l'amener à poser des actions provoquant la désapprobation des parents: «Tu es trop parfait. Et je vais t'aider à te déperfectionner » (35). La fillette amorce les leçons de désobéissance en demandant à son frère de répandre des miettes de biscuits sur le tapis du salon dans le but de contrevenir aux règles de la maison établies par la mère. Il faudra toutefois que la jeune délinquante prépare une seconde leçon pour que la désobéissance produise les résultats escomptés. Conseillé par sa sœur, Raphaël entreprend de dessiner sur les murs avec de la gouache. Il s'ensuit une escarmouche entre les contrevenants qui répandent de la peinture allègrement dans la pièce. Dès que la mère est mise au fait de l'incident, les deux enfants écopent d'une punition. Comme le souligne Annette, l'expérience a 1'avantage de rapprocher le frère et la sour: « Après l'épisode du pot de peinture renversé, on est devenus très complices [...]. On fait toutes sortes de folies. Tours au téléphone, farces, attrapes » (53). En guise d'examen final, Raphaël devra réaliser une potion magique au moyen d'ingrédients hétéroclites: « Le problème avec mon frère et moi, c'est qu'on n'arrive pas à désobéir sans faire de dégâts. Le comptoir de la cuisine est dans un tel état! » (55). La fillette rapporte que Raphaël finit par avouer toute la vérité à sa mère et redevient sage au terme de cet incident, comme quoi les paroles maternelles « on ne peut changer les gens »sont justes $(60)$. Si la mère tient pour acquis que l'identité relève de la permanence et que, par conséquent, les gens ne changent pas, ses paroles incitent la petite fille à être attentive aux perspectives de l'autre et à prendre conscience d'un monde qui n'est pas à son image. 
À l'aune d'un discours qui met en lumière la nature angélique du jeune frère, le texte exhibe les différences entre la fillette et son cadet. Ce parallélisme sert à amplifier certains traits de la personnalité de l'ainée décrite comme opiniâtre et réfractaire à l'autorité. Comme le souligne à maintes reprises le sujet féminin, Raphaël est différent de sa sœur à tous points de vue: « À son âge, je mangeais des bonbons en cachette et je détestais l'école, moi! » (25). Contrairement au bambin qui s'en remet à des " chevaliers redresseurs de torts » (10) pour imaginer le bien et vaincre le mal, la narratrice ne compte point de références de la sorte dans son imagerie: « Il [Raphaël] a des héros comme tous les enfants, sauf moi » (10). Aux héros conventionnels qui émergent d'un imaginaire fondé sur des oppositions manichéennes, Annette préfère les petites filles originales sorties tout droit des romans contemporains pour la jeunesse. De fait, la fillette considère Fifi Brindacier comme " la reine de la désobéissance » (48). Fidèle lectrice des péripéties d'une orpheline qui n'a que faire de la bienséance et des convenances, la protagoniste voue une admiration sans bornes à l'héroïne de l'œuvre romanesque dont l'écrivaine suédoise, Astrid Lindgren, est l'auteure: « Les aventures de cette fille si différente des autres sont époustouflantes » (48). Au demeurant, l'intertexte comporte une fonction herméneutique qui vient préciser le sens du texte citant : les allusions à Fifi Brindacier servent à caractériser la psyché d'une fillette s'écartant des normes sociales. Plus qu'une enfant, Fifi est un boute-en-train qui personnifie tous les désirs de l'enfance. Elle allie une irrépressible vitalité, un rêve de liberté à une révolte puissante, et retourne souvent les armes des adultes contre eux-mêmes. Au fond, elle met en question la société et ses normes. Aussi Raphaël saisit-il bien l'importance que revêt pour sa sœur le contenu de 1'ouvrage: «Il prend mon livre pour voir l'image de Fifi qui sert le café, perchée dans un arbre " (49). La scène dont il est question figure au cinquième chapitre du livre de Lindgren, lequel est intitulé " Perchés sur une barrière et dans un arbre ». Il s'agit bien entendu d'une scène révélatrice des actions farfelues que peut accomplir la célèbre héroïne: rien de plus naturel que de servir du café à des amis qui se prélassent, rivés aux branches d'un chêne. Pour ses camarades, Fifi est toujours prête à se dévouer ou à inventer des jeux extraordinaires. Toujours est-il que le jeune frère comprend la portée du bouquin au sein de l'existence d'Annette, qui privilégie à son tour les situations non conventionnelles, au moment où il affirme: « C'est ton héroïne! » (49). Une caricature réalisée par la fillette, la représentant en prisonnière auprès de son frère déguisé en policier, associe respectivement l'ordre et le désordre à l'un et à l'autre des personnages. Après la lecture du texte consacré aux aventures de Fifi, l'illustration prend tout son sens aux yeux de Raphaël. Comme le jeune garçon se familiarise avec une autre façon d'appréhender le monde à travers la réalité de Fifi Brindacier, il saisit mieux pourquoi sa sœur préfère jouer le rôle du hors-la-loi à celui d'un représentant des forces de l'ordre.

Si Fifi ne trouve pas l'école amusante, Annette ne se fait point d'illusion quant aux lieux où l'on est censé transmettre des connaissances. C'est pourquoi celle-ci tente de faire comprendre à son compagnon qu' « à l'école, on nous enseigne beaucoup de choses que l'on sait déjà " (15). À l'instar de Fifi dont 
l'imagination est débordante, la fillette se plaît à rêver. Si Raphaël « sait tout » et « est le spécialiste de tout ce qui vit dans l'univers » (18), la jeune narratrice préfère les méandres de l'imagination: « Moi, je suis toujours dans la lune et je ne suis la spécialiste de rien » (18). De fait, le gamin est passionné par la science et défend la cause écologique avec ferveur: « Il pose des questions sur les animaux en voie d'extinction " (60). À son exemple, la maisonnée doit souscrire aux pratiques visant à protéger l'environnement. Pour le contrarier, la petite fille ne suit pas les consignes: « J'ai fait couler l'eau du robinet pendant au moins cinq minutes. Il s'est mis dans une de ces colères! $\grave{A}$ croire que la survie de planète avait été menacée par moi! »(17-18). Rien d'étonnant si le jeune garçon reproche à sa sœur de ne pas compléter ses cahiers: « Ça gaspille du papier! " (61). Son zèle va jusqu'à bannir certaines émissions de télévision des soirées familiales. Préoccupé par la violence présentée au petit écran, semble-t-il, Raphaël refuse de visionner une production cinématographique mettant en scène le détective Colombo, une production qui, selon Annette, demeure inoffensive. Les scènes qui font du petit garçon un protecteur de l'environnement et un défenseur de valeurs pacifiques servent à cristalliser la pensée critique de la petite fille.

Si Annette comprend que la révolte n'est pas « naturelle » chez son cadet, elle apprend à apprécier davantage le bambin qui, lui, a bien cerné, qu'à travers les mots « on ne peut changer les gens", se dissimule une morale à leur fameuse odyssée dans l'univers de la désobéissance. Aussi, la fillette prend conscience que l'esprit de Raphaël est davantage sollicité par la forme que la portée des messages prônant l'insubordination. Lorsque la petite fille lui communique une liste de recommandations visant à le rendre plus rebelle que docile, le jeune garçon perçoit d'emblée au sein des énoncés des rimes qu'il associe à une comptine: « 1 . Quand tu vas au magasin, choisis le jouet le plus cher. 2. Au restaurant, pense tout de suite au dessert. 3. À l'école, essaie de ne pas trop en faire » (61). Malgré qu'ils réagissent différemment aux effets d'un conditionnement social, les enfants adhèrent tous deux au monde de la poésie et de la musique des mots.

\section{La petite fille et les simulacres de la nuit : la victoire de la raison}

À nouveau complices, le frère et la sceur doivent affronter les monstres de la nuit. Le discours de la petite fille établit en effet un parallélisme entre le monde diurne et le monde nocturne dans le dernier tome de la série qui s'intitule Annette et le vol de nuit. Le roman s'amorce sur une note plutôt négative puisque survient un cambriolage dès les premières lignes. Après avoir célébré le début des vacances estivales dans un restaurant italien, la famille rentre au bercail pour y trouver des lieux pillés et chambardés: « Dans le salon, des vidéocassettes étaient éparpillées sur le divan. Les livres dans la bibliothèque étaient pêle-mêle. Une fenêtre était cassée dans la cuisine " (Turcotte 2000,10). Curieusement, c'est Annette qui adopte l'attitude la plus raisonnable au beau milieu d'une situation chaotique alors qu'elle pense à appeler du secours: «Ok. On se calme ! Ai-je crié » (13). Le lendemain matin, la fillette tente d'analyser froidement les conséquences du vol perpétré à leur domicile: « Il a fallu que je leur explique qu'on ne pourrait plus jamais être tranquilles. On savait maintenant que tout pouvait arriver pendant 
notre absence » (29). Contrairement à ce que pense le policier, la petite fille a la certitude que le cambrioleur va récidiver. C'est pourquoi elle accuse sa mère et son frère de réagir aux circonstances avec désinvolture: « Vous n'avez pas l'air de prendre ça au sérieux » (28). En observant attentivement l'aînée, l'héroïne finit par comprendre que cette dernière s'efforce de sourire pour ne pas inquiéter ses enfants et que, par conséquent, les apparences peuvent être trompeuses.

Bien qu'elle se définisse comme une "force » capable de calmer les esprits échauffés, la jeune narratrice est profondément troublée par les événements. Terrifiée à l'idée que le voleur puisse revenir, la gamine ne ferme pas l'œil de la nuit tandis qu'elle imagine le pire et voit les objets de sa chambre arborer des formes étranges: « Je commençais à avoir la frousse. Je fabulais à propos de tout ce qui pouvait se cacher dans un placard » (19). La petite fille est consciente du fait que la peur peut altérer la perception des êtres et des choses: « Dans la pénombre, ma chambre paraissait différente. 11 y avait des ombres chinoises sur le mur » (20). Ainsi se dessine à travers le récit à la première personne une opposition entre la fiction et la réalité qu'un vocabulaire relié à la défiguration opérée par une conscience perceptive incarne avec brio: " on aurait dit qu'elle [la porte de la garde-robe] bougeait »; " cela ressemblait à des froissements d'ailes »; " chaque objet était en train de se transformer »; « mes poupées s'allongeaient »; " les pages de mes cahiers tressaillaient "; « les vêtements sur ma chaise avaient une forme humaine » (20-21). Si le sujet féminin conçoit que l'objet de sa perception peut se réduire à des simulacres provoqués par « la peur de la nuit » (22), il n'en demeure pas moins que le produit de son imagination le confond.

Toujours est-il que les phantasmes ne prennent pas le dessus sur la raison d'Annette. Bien décidée à surmonter son traumatisme et à isoler du réel des mondes construits par une imagination galvanisée par la peur, la fillette propose un plan à son frère. Il s'agit de passer la nuit dehors à faire le guet afin de démasquer le cambrioleur qui a saccagé leur résidence: « J'avais décidé de nous transformer, Raphaël et moi, en veilleurs de nuit. Je voulais affronter et combattre ma peur » (33-34). Pareille initiative montre que l'héroïne refuse de se conformer à une situation où elle doit assumer un rôle de victime. Au lieu de passer une autre nuit à appréhender le retour du voleur et à craindre les fantômes qui envahissent sa chambre, la petite fille souhaite se fondre à la réalité des ténèbres en compagnie de Raphaël. L'expérience pour le moins inusitée engendre un niveau de compréhension de la réalité et des idées reçues qui la façonnent, si bien que la protagoniste saisit dans l'obscurité le sens d'un proverbe bien connu: « La nuit, tous les chats sont gris. J'ai vite compris ce que ce proverbe signifie " (41). En se promenant dans le noir, Annette a l'impression que les lieux pourtant familiers ne revêtent plus la même allure: « Les maisons avaient changé de couleur. Les arbres méditaient. On aurait juré que nous étions dans une autre ville. Et nous, surtout, nous avions l'air plus petits "(39). Forte de cette découverte, la fillette s'arrange pour inverser l'ordre des choses: " Rendus au parc, nous avons grimpé tous les deux au sommet de la fusée géante. Ainsi, nous dominions ce monde inconnu » (39). Mais ce sentiment de puissance est éphémère puisque les enfants sont interrompus par la présence d'un homme que la petite fille compare à « un ogre dans 
un conte de fées », lorsque celui-ci prononce: « Est-ce que je vois bien là deux petits enfants? » (59). Au moment où il s'approche des petits et les interpelle, le noctambule leur cause une peur bien réelle. Pourtant, l'homme ne poursuit pas les « veilleurs de nuit » qui s'enfuient à toutes jambes. À la suite de l'expérience vécue au parc, Annette modifie ses croyances et sa conception de l'obscurité qu'elle associe désormais à l'émergence de mondes possibles dont la prise de conscience s'acquiert au moyen d'une activité perceptive: « Il y avait donc tout un peuple d'animaux qui ne sortaient que la nuit! » (43-44). Au même titre que l'expérience sensible permet de transformer la signification que l'on prête aux objets, la vie de la nuit a fait découvrir d'autres mondes au personnage féminin. Aussi la gamine fait-elle sien le célèbre axiome « Tout est relatif du point de vue de l'observateur », alors que son périple l'amène à conclure à son tour: « Tout dépend de la façon dont on regarde les choses » (Turcotte 2000, 56). En optant pour l'expérience, la protagoniste a modifié sa façon de penser à propos de la matière, de l'espace et du temps. Ce faisant, elle apprend à distinguer le monde des apparences de la réalitéviii. Si la nuit à la belle étoile a été pour la fillette une source de frayeur, elle lui aura permis de vaincre d'autres appréhensions: « Au moins, maintenant, les ombres de ma chambre ne me feraient plus jamais peur » (56). Au lieu de craindre la venue des cambrioleurs et de se poser en victime malheureuse, l'héroïne fait l'apprentissage de l'une des multiples réalités de la nuit.

Toujours à l'affût d'événements à transposer dans ses cahiers, Annette s'empresse d'écrire le récit de sa nuit mouvementée. Cependant, l'émotion est telle que la petite fille n'arrive pas à la coucher sur le papier: « Dès que je les attrapais, les mots s'envolaient comme des papillons » (56). Non seulement la protagoniste est-elle incapable de se concentrer après sa mésaventure au parc, mais elle ne peut poursuivre ses lectures lorsqu'elle se met au lit à la suite du cambriolage. Tout se passe comme si la peur de la nuit paralysait la jeune lectrice. Il importe de noter que la fillette a pour livre de chevet un roman de la comtesse de Ségur: « J'ai allumé ma lampe de chevet pour lire Pauvre Blaise » (17). L'allusion indique que la narratrice est familière avec l'œuvre ségurienne et ses personnages. Paru en 1862, Pauvre Blaise raconte l'histoire d'un garçon victime de la méchanceté d'un autre. Conformément à l'imaginaire développé dans les textes de l'écrivaine, Pauvre Blaise incarne un monde manichéen où s'affrontent le bien et le mal personnifiés, en l'occurrence, par Blaise et Jules. Si ce dernier est le fils d'un riche comte habitant un château, Blaise est issu d'une famille modeste, son père exerçant le métier de concierge. L'un des traits qui caractérise Blaise, ce fils de paysan soumis à la volonté d'un garçon égoïste et sans scrupules, est sans contredit sa résignation et son esprit de sacrifice. De toute évidence, la figure du personnage masculin s'oppose à celle d'Annette qui, loin de se résigner et d'attendre l'intervention d'une force divine pour modifier le cours des événements, se définit par l'action et une pensée critique. Encore une fois, les renvois au texte cité servent, par ricochet, à décrire le personnage féminin du texte citant. 
La représentation de la petite fille dans la série pour la jeunesse d'Élise Turcotte ne souscrit guère aux modèles féminins de soumission et de passivité mis de l'avant dans l'œuvre de la comtesse de Ségur. Issue d'une famille dont les parents sont séparés, Annette qui évolue avec sa mère et son jeune frère dans un milieu urbain incarne à bien des égards un mode de vie moderne marqué par la fin des certitudes. Au sein de ses cahiers, la fillette fait l'expérience de la signification et de la relation à travers le langage, dans la mesure où elle s'intéresse aux mécanismes de la pensée. Alors qu'elle s'adonne à la lecture et à l'écriture, elle prend conscience de l'unicité d'un moi qui demande à s'énoncer de façon particulière. Admirant les héroïnes insoumises, elle songe à enseigner la désobéissance à Raphaël, son jeune frère, pour l'écarter des conventions sociales. Bien qu'elle soit confrontée à une situation qui la bouleverse, l'héroïne refuse de jouer le rôle de la victime. Si elle privilégie l'action et l'expérience qui la détermine, elle arrive à surmonter les peurs émanant de sa naïveté. Quoiqu'elle mette en question le discours et les actions d'une mère qu'elle juge trop conventionnelle, elle est en mesure de pratiquer l'autocritique et de reconnaître ses torts quand elle va trop loin. Réfractaire au savoir véhiculé à l'école, Annette est une petite fille dont l'imaginaire se nourrit d'abord de l'expérience quotidienne et de nombreuses lectures. Dans le prolongement des pratiques féministes, la fillette s'écarte des stéréotypes et des rôles sexués, si bien qu'elle cherche à façonner son moi social au moyen d'une pensée autonome. À l'instar d'Annette, plusieurs petites filles prennent la parole et développent des imaginaires valorisant une pensée créatrice, en rupture avec les idées reçues du patriarcat, au sein de la production romanesque pour la jeunesse de la dernière décennie. Sans doute serait-il opportun de procéder à l'analyse de l'ensemble de ces pratiques discursives émanant d'une écriture qui contribue à représenter les fillettes comme de véritables sujets.

\section{Notes}

${ }^{i} \mathrm{C}$ 'est à tout le moins le questionnement qui amène Anna $\mathrm{Y}$. Sumida à interpréter le texte d'une fillette de six ans: « Living in a postmodern world, at the brink of the new millenium, would a girl of the new age view herself as passive or liberated ? Empowered or powerless? Equal or unequal to males? » (309).

ii Dans une étude portant sur la réussite scolaire des filles au primaire, Claudette Gagnon examine les rapports sociaux de sexe et en arrive à cette conclusion: «Chez les filles, il y a opposition aux étiquettes stéréotypées. Cette opposition remet en question la culture dite féminine qui les enferme dans des rôles stéréotypés. [...] Ce n'est pas que la culture féminine soit inexistante; elle vise tout simplement à l'émancipation des rôles sociaux traditionnels » (33).

iii Vinson appuie sa démonstration sur les travaux de Michel Foucault.

iv Comme en font état les orientations éditoriales de la Courte échelle, la collection « Premier roman » s'adresse aux lecteurs en apprentissage et comporte des textes brefs. La simplicité du vocabulaire, l'humour, la richesse des personnages et les illustrations garantissent l'intérêt du jeune lectorat. 
' Il s'agit des protagonistes principales des Petites filles modèles, que 1'on retrouve également dans Les malheurs de Sophie et Les vacances.

vi On reconnaît les grands discours qui ont façonné la pensée occidentale. Selon une approche féministe, le métarécit se définit bien entendu comme patriarcal.

vii Malik Allam décrit les types de situation qui donnent lieu à la pratique de l'écriture intime (116).

viii Comme 1'explique Philippe Malrieu, « [simulacres] et fictions sont deux aspects importants de la socialisation de l'enfant » (177).

\section{Bibliographie}

Allam, Malik. Journaux intimes. Sociologie de l'écriture personnelle. Paris: L'Harmattan, coll. « Logiques sociales », 1996.

Belotti, Elena G. Du côté des petites filles. Paris: Éditions des femmes, 1974.

Boisvert, Nicole M. Le mensonge de Myralie. Montréal: Éditions Michel Quintin, coll. " Nature jeunesse », 1999.

Chevalier, Jean et Alain Gheerbrant. Dictionnaire des symboles. Mythes, rêves, coutumes, gestes, formes, figures, couleurs, nombres. Paris: Robert Laffont, 1982. Cromer, Sylvie et Adela Turin. «Que racontent les albums illustrés aux enfants? Ou comment présente-t-on les rapports hommes-femmes aux plus jeunes ? 》. Recherches féministes 11:1 (1998). 223-230.

Demers, Dominique. Marie la chipie. Montréal: Québec/Amérique, coll. «Bilbo », 1997.

Foucault, Michel. Surveiller et punir. Naissance de la prison. Paris: Gallimard, coll. « Bibliothèque des histoires », 1975.

Gagnon, Claudette. « La dynamique de la réussite scolaire des filles au pri maire: les motivations et les enjeux des rapports sociaux de sexe ». Recherches féministes 11:1 (1998). 19-45.

Hébert, Marie-Francine. Un monstre dans les céréales. Montréal: La Courte échelle, coll. « Premier roman », 1988.

Leblanc, Louise. Sophie lance et compte. Montréal: La Courte échelle, coll. « Premier roman », 1991.

Lindgren, Astrid. Fifi Brindacier. Paris: Hachette, 2001 [1945].

Malrieu, Philippe. La construction des imaginaires. Montréal: L'Harmattan, coll. "Psychanalyse et civilisations », 2000.

Marineau, Michèle. Cassiopée ou l'été polonais. Montréal: Québec/Amérique, coll. " Littérature jeunesse ", 1988.

Rey, Alain et J. Rey-Debove. Le Petit Robert I. Dictionnaire alphabétique et analogique de la langue française. Paris: Le Robert, 1987.

Ségur, Sophie de. Pauvre Blaise. Paris: Casterman, 1971 [1862].

L Les Malheurs de Sophie. Paris: Casterman, 1979 [1860].

Les Vacances. Paris: Casterman, 1974 [1859].

Les Petites filles modèles. Paris: Casterman, 1979 [1858]. 